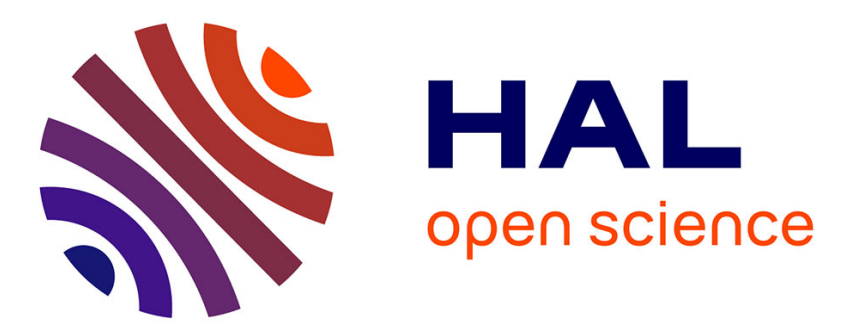

\title{
Simple modeling of energy consumption for D2D relay mechanism
}

Cesar Vargas Anamuro, Nadège Varsier, Jean Schwoerer, Xavier Lagrange

\section{To cite this version:}

Cesar Vargas Anamuro, Nadège Varsier, Jean Schwoerer, Xavier Lagrange. Simple modeling of energy consumption for D2D relay mechanism. Wireless Communications and Networking Conference Workshops, Apr 2018, Barcelone, Spain. hal-01836546

\section{HAL Id: hal-01836546 https://imt-atlantique.hal.science/hal-01836546}

Submitted on 12 Jul 2018

HAL is a multi-disciplinary open access archive for the deposit and dissemination of scientific research documents, whether they are published or not. The documents may come from teaching and research institutions in France or abroad, or from public or private research centers.
L'archive ouverte pluridisciplinaire HAL, est destinée au dépôt et à la diffusion de documents scientifiques de niveau recherche, publiés ou non, émanant des établissements d'enseignement et de recherche français ou étrangers, des laboratoires publics ou privés. 


\title{
Simple modeling of energy consumption for D2D relay mechanism
}

\author{
Cesar Vargas Anamuro*, Nadège Varsier*, Jean Schwoerer* and Xavier Lagrange ${ }^{\dagger}$ \\ *Orange Labs, Meylan, France \\ Email: \{cesaraugusto.vargasanamuro, nadege.varsier, jean.schwoerer\}@orange.com \\ ${ }^{\dagger}$ IMT Atlantique/IRISA, Rennes, France \\ Email: xavier.lagrange@imt-atlantique.fr
}

\begin{abstract}
Reducing energy consumption is a key requirement for Massive Machine-Type Communication (mMTC) devices operating on battery power. Device-to-Device (D2D) communication is a promising technology that can be used in $5 \mathrm{G}$ networks for this purpose. In this paper, we identify the interest of using a D2D relay mechanism compared with a direct link with an unfavorable link budget. We first look for an optimal Relay-MTC device configuration that minimizes the energy consumption. We then compare the energy consumption in direct and relaying mode expressing it in terms of the distance between the base station and the MTC device and between the base station and the relay. We show that when the MTC device is close to the base station, D2D relay mechanism can reduce the energy consumed by the MTC device at the expense of an increase in the global energy consumption (i.e. the energy consumed by the MTC device plus the energy consumed by the relay). On the other hand, when the MTC device is far from the base station, D2D relay mechanism allows reducing both the energy consumption by the MTC device and the global energy consumption.
\end{abstract}

Index Terms-Energy consumption, Device-to-Device, MTC

\section{INTRODUCTION}

In the coming years, the number of connected devices will grow exponentially [1], many of these devices being MachineType Communication (MTC) devices. MTC applications can be grouped into two main categories [2]: critical MTC and massive MTC. Critical MTC applications are characterized by very low latency, very high reliability and availability. On the other hand, massive MTC (mMTC) applications are characterized by a large number of devices, low mobility, small and infrequent data transmission, long battery life, low device cost, and low complexity.

Cellular networks will play an important role in the deployment of MTC applications due to their characteristics, e.g. scalability, reliability, large coverage, security, dedicated spectrum, and simplicity of management [3]. However, using cellular networks for mMTC applications would lead to network congestion and increased cost of deep indoor coverage. Device-to-Device (D2D) is a promising technology that aims to connect two or more devices directly without going through a direct link between the MTC device (MTD) and the Base Station (BS). D2D relaying can reduce the energy consumption, extend the network coverage and have a more efficient use of radio resources [4].

An MTD operating on battery power requires low-energy consumption, since the battery replacement in this device is a difficult task or even an impossible task in some cases. To answer to this low-energy consumption need, in [5] [6] the authors propose to group terminals in clusters in which direct communication is possible. A particular terminal acts as a cluster head which has the function of a local coordinator and data forwarding. The other cluster members send their data to the cluster head. Certainly, clustering approach is an interesting method to reduce the energy consumption in MTDs. However, the authors analyze the formation of clusters and the selection of cluster-heads considering that all devices have similar characteristics. In this paper, we focus on using relaying mechanisms, considering that a device could be a relay or a cluster-head only if it has better characteristics than an ordinary MTD (e.g. better battery life) since relays may experience major burden in energy consumption. Thus, we consider that a User Equipment (UE) acts as a relay helping in the connection of MTDs with the base station. Also, MTDUE links are established through a D2D communication as in [7] [8] [9].

We first define an energy consumption model. There are many proposed techniques in the literature for modeling the energy consumption [6] [10] [11]. In [6] a detailed energy consumption model for clustering is presented. In [10] they propose a realistic model for multi-hop networks and power consumption model for wireless sensor devices in [11]. In addition, 3rd Generation Partnership Project (3GPP) specifications suggest an energy consumption evaluation methodology in [12]. The model used in this work is inspired by the models proposed in [6] and [12], but focus only on the transmission phase.

One of our main objectives is to identify criteria for establishing the interest of using D2D relay mechanisms, looking for the optimal configuration minimizing the energy consumption. In [13], the authors have defined the energy consumption in WiFi-D2D and LTE-D2D as a function of the packet size and the number of devices in clustering MTDs. In [14], the authors discuss conditions under which a D2D communication is preferable to a communication via the $\mathrm{BS}$ in dynamic Time-Division Duplex (TDD) systems. In our study we compare the energy consumption in direct and relaying mode expressing it as a function of the distances between the BS and the MTD and between the BS and the relay.

The paper is organized as follows. In section II, we present 
the scenario and system model. In Section III, we look for the minimum energy consumption in relaying mode. In Section IV, we compare the energy consumption in direct and relaying mode. In Section V, we present simulation results and compare them with derived analytical expressions from sections III and IV. Finally, we conclude in Section VI.

\section{SCENARIO AND SYSTEM MODEL}

\section{A. Scenario and hypotheses}

We consider a coverage scenario where both relays and MTDs are in-coverage. In this case, an MTD could connect to the network in two ways: directly to the BS or using a relay. In order to build a simplified model the following assumptions are made:

- Cellular infrastructure supports the discovery and synchronization phases (Network assisted approach). Thus, all devices are synchronized with the BS. We analyze only the energy consumption in data transmission phase.

- MTDs receive the data directly from the BS, i.e. downlink without using a relay.

- Relays are devices that could help MTDs to connect to the network, for which they receive and retransmit the packets coming from MTDs. Our analysis is focused on uplink since MTC applications mainly generate traffic in uplink.

- Relays are connected to the network using cellular links and MTDs are connected to the relays using D2D links. D2D links and cellular links are orthogonal, there is no interference between these systems.

- The communication channel is well known by devices. That means a perfect dynamic adaptation of the modulation and coding scheme.

- Devices transmit at their maximum allowed transmission power, there is no power control.

\section{B. Channel Model}

In order to simplify our analysis, we model the communication channel only considering path loss. According to the Okumura-Hata model, the received power $P_{r x}$ can be derived as

$$
P_{r x}=P_{t x} \frac{K}{d^{\gamma}},
$$

where $P_{t x}$ is the transmission power, $K$ is the constant path loss factor, $d$ is the distance between the transmitter and the receiver, and $\gamma$ is the path loss exponent.

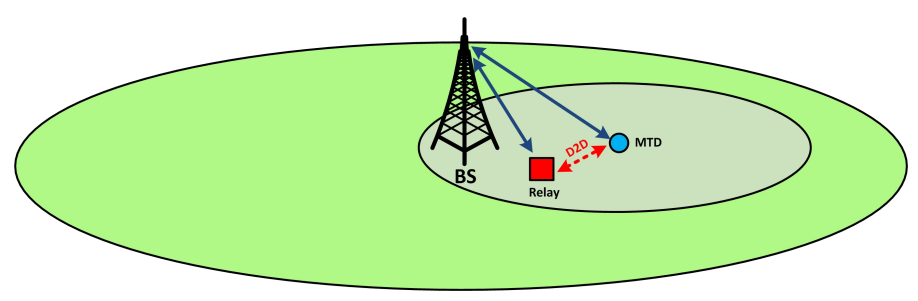

Fig. 1. Coverage scenario.

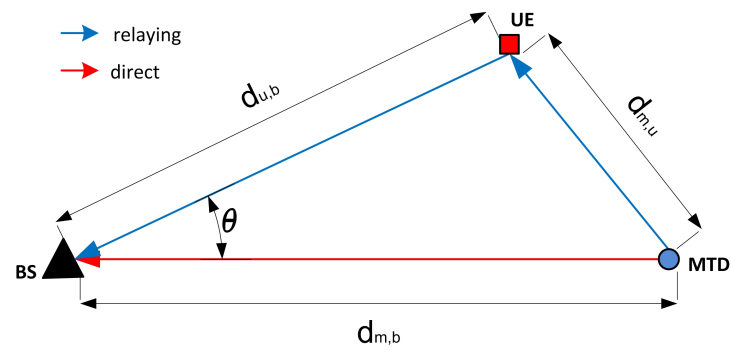

Fig. 2. Transmission scheme in direct and relaying mode.

\section{Link Model}

We consider a low load situation. Hence the interference is negligible. Also, we consider only additive white Gaussian Noise (AWGN). From Shannon's capacity equation, the data rate $R$ is derived as

$$
R=W \log _{2}(1+\eta),
$$

where $W$ is the transmission bandwidth, and $\eta$ is the Signal-toInterference-and-Noise-Ratio (SINR) which can be expressed as $\eta=P_{r x} / N_{0} W$, where $N_{0}$ is the noise power spectral density, and $P_{r x}$ is derived in (1). Note that

$$
\begin{aligned}
R & \approx W \eta \log _{2}(e), \text { when } \eta \approx 0, \\
R & \approx W \log _{2}(\eta), \text { when } \eta \gg 1 .
\end{aligned}
$$

Hereafter we consider $\eta \gg 1$, then the channel capacity can be rewritten as

$$
R_{i, j}=W_{i} \log _{2}\left(\frac{K P_{i}}{N_{0} W_{i} d_{i, j}^{\gamma}}\right),
$$

where $W_{i}$ and $P_{i}$ are respectively the bandwidth and the transmission power of the transmitter $i, d_{i, j}$ is the distance between the transmitter $i$ and the receiver $j$. In order to simplify the mathematical analysis, we consider the same path loss parameters $(K$ and $\gamma$ ) in all links. However, cellular communications and D2D communications have different path loss parameters in a realistic scenario.

\section{Energy Consumption Model}

We study two transmission modes: when an MTD uses a direct transmission to the BS (direct mode) and when it uses a relaying mechanism (relaying mode) as shown in Fig. 2. A device can operate in one of the following states: Tx, Rx, Idle, or Sleep. However, we analyze only the energy consumption when the device is in Tx state or Rx state, since in this research work we use a simplified energy consumption model considering only the transmission phase.

a) Energy consumption in direct mode: The MTD energy consumption in direct mode can be defined as

$$
E_{m, d i r}=P_{m, T} t_{m, T}+P_{m, R} t_{m, R},
$$

where $P_{m, T}$ and $P_{m, R}$ are the MTD power consumption in Tx state and Rx state respectively, and $t_{m, T}$ is the transmission time. After transmitting its data, the MTD remains in Rx 


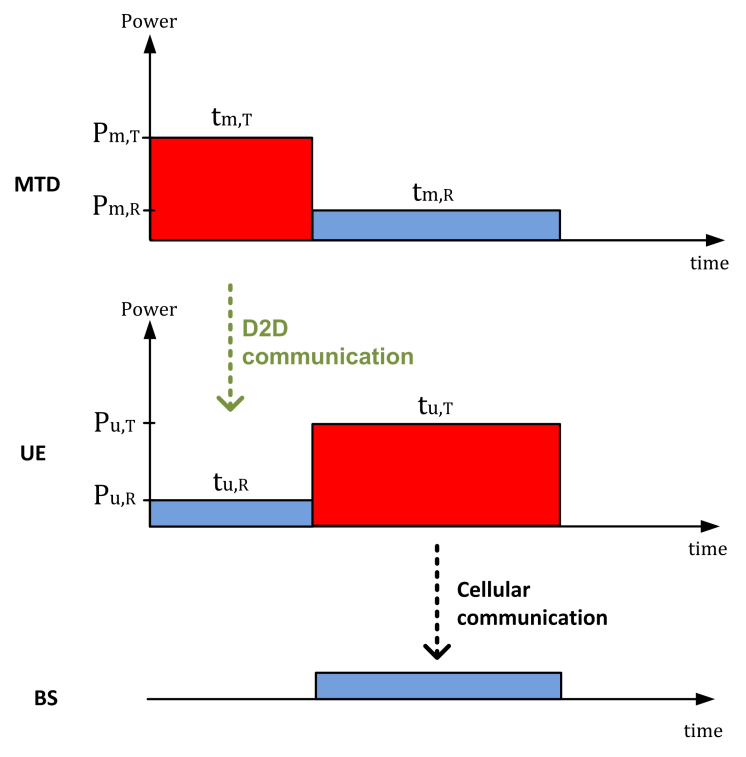

Fig. 3. Relaying process.

state a time $t_{m, R}=t_{m, T}$ waiting for some other instruction or an acknowledgement (ACK) from the BS. We have $t_{m, T}=L / R_{m, b}$, where $L$ is the packet size, and $R_{m, b}$ is the MTD transmission rate in the $M T D \rightarrow B S$ link. $R_{m, b}$ can be derived from (5) as

$$
R_{m, b}=W_{m} \log _{2}\left(\frac{K P_{m}}{N_{0} W_{m} d_{m, b}^{\gamma}}\right),
$$

where $W_{m}$ is the MTD bandwidth, $P_{m}$ is the MTD transmission power, and $d_{m, b}$ is the distance between the MTD and the BS. Then, we obtain an expression for the MTD energy consumption in direct mode:

$$
E_{m, d i r}=\frac{\left(P_{m, T}+P_{m, R}\right) L}{R_{m, b}} .
$$

b) Energy consumption in relaying mode: The total energy consumption in relaying mode $\left(E_{T, r e l}\right)$ can be calculated as the sum of the energy consumed by each device that participates in a relaying process:

$$
E_{T, r e l}=E_{m, r e l}+E_{u, r e l},
$$

where $E_{m, r e l}$ and $E_{u, r e l}$ are the energy consumption by the MTD and UE respectively. In a relaying process, the MTD first transmits its data to the UE while the UE remains in Rx state receiving and demodulating. Then, the UE retransmits the data arriving from the MTD to the BS while the MTD stays listening (Rx state) as shown in Fig. 3. In this figure we can see that $t_{m, T}=t_{u, R}$ and $t_{m, R}=t_{u, T}$.

The MTD energy consumption in relaying mode can be derived as

$$
E_{m, r e l}=P_{m, T} t_{m, T}+P_{m, R} t_{m, R},
$$

where $P_{m, T}$ and $P_{m, R}$ are the MTD power consumption in Tx state and Rx state respectively, $t_{m, T}$ and $t_{m, R}$ are the duration of Tx state and Rx state respectively. We have $t_{m, T}=L / R_{m, u}$ and $t_{m, R}=t_{u, T}=L / R_{u, b}$, then

$$
E_{m, r e l}=\frac{P_{m, T} L}{R_{m, u}}+\frac{P_{m, R} L}{R_{u, b}} .
$$

On the other hand, the UE energy consumption in relaying mode can be derived as

$$
E_{u, r e l}=P_{u, T} t_{u, T}+P_{u, R} t_{u, R},
$$

where $P_{u, T}$ and $P_{u, R}$ are the UE power consumption in Tx state and Rx state respectively, $t_{u, T}$ and $t_{u, R}$ are the duration of Tx state and Rx state respectively. We have $t_{u, T}=L / R_{u, b}$ and $t_{u, R}=t_{m, T}=L / R_{m, u}$, then

$$
E_{u, r e l}=\frac{P_{u, T} L}{R_{u, b}}+\frac{P_{u, R} L}{R_{m, u}} .
$$

Finally, we obtain the global energy consumption in relaying mode substituting (11) and (13) in (9):

$$
E_{T, r e l}=\frac{\left(P_{u, T}+P_{m, R}\right) L}{R_{u, b}}+\frac{\left(P_{m, T}+P_{u, R}\right) L}{R_{m, u}},
$$

where

$$
\begin{aligned}
R_{u, b} & =W_{u} \log _{2}\left(\frac{K P_{u}}{N_{0} W_{u} d_{u, b}^{\gamma}}\right), \\
R_{m, u} & =W_{m} \log _{2}\left(\frac{K P_{m}}{N_{0} W_{m} d_{m, u}^{\gamma}}\right) .
\end{aligned}
$$

\section{Minimum-Energy Consumption In RELAYing MODE}

In this part of the study, we look for the UE location minimizing the global energy consumption in relaying mode. Without loss of generality, we consider a BS at the origin of coordinates $(0,0)$, an MTD at a fixed position $d_{m, b}$ on the $\mathrm{x}$-axis, and a UE located anywhere with polar coordinates $\theta \in[0,2 \pi)$ and $d_{u, b} \in\left(0, R_{\text {cell }}\right]$ (see Fig. 2).

First, we normalize the distance UE-BS:

$$
d_{u, b}=x d_{m, b} .
$$

Then, the distance MTD-UE can be calculated as

$$
d_{m, u}=d_{m, b} \sqrt{x^{2}+1-2 x \cos (\theta)} .
$$

In order to simplify our analysis, let:

$$
\begin{array}{ll}
A_{1}=\frac{\left(P_{u, T}+P_{m, R}\right) L}{\gamma W_{u}}, & B_{1}=\frac{1}{\gamma} \log _{2}\left(\frac{K P_{u}}{N_{0} W_{u}}\right) \\
A_{2}=\frac{\left(P_{m, T}+P_{u, R}\right) L}{\gamma W_{m}}, & B_{2}=\frac{1}{\gamma} \log _{2}\left(\frac{K P_{m}}{N_{0} W_{m}}\right)
\end{array}
$$

Finally, the global energy consumption in relaying mode (14) becomes

$$
E_{T, r e l}=\frac{A_{1}}{B_{1}-\log _{2}\left(d_{u, b}\right)}+\frac{A_{2}}{B_{2}-\log _{2}\left(d_{m, u}\right)} .
$$

$E_{T, r e l}$ has two variables, $\theta$ and $x$. Then, to minimize the energy consumption, we calculate the partial derivatives with respect to $\theta$ and $x$ and set it equal to zero searching for a minimum. 
a) Partial derivative with respect to $\theta$ :

$$
\frac{\partial E_{T, r e l}}{\partial \theta}=\frac{A_{2} x d_{m, b}^{2} \sin (\theta)}{\ln (2) d_{m, u}^{2}\left(B_{2}-\log _{2}\left(d_{m, u}\right)\right)^{2}} .
$$

Searching for the critical points, we set $\frac{\partial E_{T, r e l}}{\partial \theta}=0$, then we have $\theta=\{0, \pi\}$. Analyzing these values, $E_{T, r e l}$ is a minimum when $\theta=0$ and is a maximum when $\theta=\pi$. Thus, the global energy consumption is minimal when BS, UE, and MTD are aligned. Also, the UE cannot be further away than the MTD $(x<1)$ since we search to minimize the global energy consumption. From (18), we can derive $d_{m, u}$ for $\theta=0$ :

$$
d_{m, u}=(1-x) d_{m, b} .
$$

Using (17) and (23) in (21) we have

$$
E_{T, r e l}=\frac{A_{1}}{B_{1}-\log _{2}\left(x d_{m, b}\right)}+\frac{A_{2}}{B_{2}-\log _{2}\left((1-x) d_{m, b}\right)} .
$$

b) Searching for the global minimum: In order to simplify our analysis, we consider $A_{2}=A_{1}$ and $B_{2}=B_{1}$. In other words, both the MTD and the UE transmit at the same power $\left(P_{m}=P_{u}\right)$, use the same bandwidth $\left(W_{m}=W_{u}\right)$ and consume the same power when they are in the same state $\left(P_{m, T}=P_{u, T}\right.$ and $\left.P_{m, R}=P_{u, R}\right)$. Then, substituting in (24) we have

$$
E_{T, r e l}=\frac{A_{1}}{B_{1}-\log _{2}\left(x d_{m, b}\right)}+\frac{A_{1}}{B_{1}-\log _{2}\left((1-x) d_{m, b}\right)} .
$$

Using the first partial derivative criteria, we can acquire the critical points solving $\frac{\partial E_{T, r e l}}{\partial x}=0$. Thus we have

$$
(1-x)\left(B_{1}-\log _{2}\left((1-x) d_{m, b}\right)\right)^{2}-x\left(B_{1}-\log _{2}\left(x d_{m, b}\right)\right)^{2}=0,
$$

we can find a critical point at $x=0.5$. To determine if this point is a local minimum or a local maximum, we have to calculate the second partial derivative with respect to $x$ and evaluate it at $x=0.5$ :

$$
\left.\frac{\partial^{2} E_{T, r e l}}{\partial x^{2}}\right]_{x=0.5}=\frac{8 A_{1}\left(\log _{2}\left(e^{2} d_{m, b}\right)-B_{1}-1\right)}{\ln (2)\left(B_{1}+1-\log _{2}\left(d_{m, b}\right)\right)^{3}} .
$$

Using the second derivative criteria, if expression (27) is negative then $E_{T, r e l}$ is concave around $x=0.5$ (i.e. a local maximum). On the contrary, if expression (27) is positive then $E_{T, r e l}$ is convex around $x=0.5$ (i.e. a local minimum). We know that $A_{1}>0$ and when $\eta \gg 1$, so it can be deduced that $B_{1}>\log _{2}\left(d_{m, b}\right)$. Therefore, in (27), $\left.E_{T, r e l}\right]_{x=0.5}$ is a local minimum when

$$
\begin{gathered}
\log _{2}\left(e^{2} d_{m, b}\right)-B_{1}-1>0, \\
d_{m, b}>2^{B_{1}+1} / e^{2} .
\end{gathered}
$$

From (19), $B_{1}=\frac{1}{\gamma} \log _{2}\left(\frac{K P_{u}}{N_{0} W_{u}}\right)$ then we have

$$
d_{m, b}>\frac{2}{e^{2}}\left(\frac{K P_{u}}{N_{0} W_{u}}\right)^{1 / \gamma} \text {. }
$$

In conclusion, $\left.E_{T, r e l}\right]_{x=0.5}$ is a local minimum when condition (30) is satisfied, otherwise it is a local maximum.

Condition (30) only ensure a local minimum at $x=0.5$ depending on the distance $d_{m, b}$. However, we search for the point where $E_{T, r e l}$ is the global minimum, for which we compare all possible local minima and determine which one is the global minimum. $E_{T, \text { rel }}$ may have three local minima at $x=0, x=0.5$ and $x=1$, but by symmetry $\left.\left.E_{T, r e l}\right]_{x=0}=E_{T, r e l}\right]_{x=1}$. Therefore, we search for the global minimum comparing $\left.E_{T, r e l}\right]_{x=0.5}$ and $\left.E_{T, r e l}\right]_{x=1}$.

Using expression (25) at $x=0.5$ we have

$$
\left.E_{T, r e l}\right]_{x=0.5}=\frac{2 A_{1}}{B_{1}+1-\log _{2}\left(d_{m, b}\right)} .
$$

$\left.E_{T, r e l}\right]_{x=1}$ is obtained also using expression (25). Physically, $x=1$ means that MTD and UE are in the same location. In other words, the receiving power must be equal to the transmission power in the MTD-UE link. Therefore, $\lim _{x \rightarrow 1}\left(B_{1}-\log _{2}\left((1-x) d_{m, b}\right)\right)=\frac{1}{\gamma} \log _{2}\left(\frac{P_{m}}{N_{0} W_{m}}\right)$, in (25):

$$
\left.E_{T, r e l}\right]_{x=1}=\frac{A_{1}}{B_{1}-\log _{2}\left(d_{m, b}\right)}+\frac{A_{1}}{C},
$$

where

$$
C=\frac{1}{\gamma} \log _{2}\left(\frac{P_{m}}{N_{0} W_{m}}\right) .
$$

$\left.E_{T, r e l}\right]_{x=0.5}$ is the global minimum when:

$$
\left.\left.E_{T, r e l}\right]_{x=0.5}<E_{T, r e l}\right]_{x=1} \text {. }
$$

Substituting (31) and (32) in (34), we have

$$
\frac{2 A_{1}}{B_{1}+1-\log _{2}\left(d_{m, b}\right)}<\frac{A_{1}}{B_{1}-\log _{2}\left(d_{m, b}\right)}+\frac{A_{1}}{C} .
$$

Expression (35) can be rewritten as

$$
Q\left(\log _{2}\left(d_{m, b}\right)\right)>0
$$

where

$$
Q(y)=y^{2}+\left(C-2 B_{1}-1\right) y+B_{1}^{2}+B_{1}-C B_{1}+C .
$$

We estimate the discriminant of $Q(y)$

$$
\Delta=C^{2}-6 C+1 .
$$

If $\Delta<0$, then condition (36) is always satisfied for any value of $d_{m, b}$. If $\Delta>0$, then it is satisfied for

$$
d_{m, b}<2^{\frac{2 B_{1}+1-C-\sqrt{\Delta}}{2}} \text { or } d_{m, b}>2^{\frac{2 B_{1}+1-C+\sqrt{\Delta}}{2}} .
$$

From (19), $B_{1}=\frac{1}{\gamma} \log _{2}\left(\frac{K P_{u}}{N_{0} W_{u}}\right)$ then

$$
d_{m, b}<\sqrt{2}\left(\frac{K P_{u}}{N_{0} W_{u}}\right)^{\frac{1}{\gamma}} 2^{\frac{-C-\sqrt{\Delta}}{2}}
$$

or

$$
d_{m, b}>\sqrt{2}\left(\frac{K P_{u}}{N_{0} W_{u}}\right)^{\frac{1}{\gamma}} 2^{\frac{-C+\sqrt{\Delta}}{2}} .
$$

In conclusion, if $\Delta<0$, then the global minimum is always $\left.E_{T, r e l}\right]_{x=0.5}$ for any value of $d_{m, b}$. If $\Delta>0$, then $\left.E_{T, r e l}\right]_{x=0.5}$ is the global minimum only if condition (40) or (41) is satisfied. Otherwise, the global minimum is $\left.E_{T, r e l}\right]_{x=1}$. Note that, $\Delta>0$ if $C<3-2 \sqrt{2}$ or $C>3+2 \sqrt{2}$. 


\section{COMPARISON OF ENERGY CONSUMPTION IN DIRECT MODE AND RELAYING MODE}

In this section, we compare the minimum energy consumption in relaying mode and the energy consumption in direct mode. We can rewrite expression (8) as

$$
E_{m, d i r}=\frac{A_{0}}{B_{0}-\log _{2}\left(d_{m, b}\right)},
$$

where $A_{0}=\frac{\left(P_{m, T}+P_{m, R}\right) L}{\gamma W_{m}}$ and $B_{0}=\frac{1}{\gamma} \log _{2}\left(\frac{K P_{m}}{N_{0} W_{m}}\right)$.

$\min \left(E_{T, r e l}\right)$ may be $\left.E_{T, r e l}\right]_{x=1}$ or $\left.E_{T, r e l}\right]_{x=0.5}$ depending on $d_{b, m}$ as we have determined in section III. By doing a simple analysis, we conclude that $\left.E_{T, r e l}\right]_{x=1}$ is never less than $E_{m, d i r}$. Therefore, we analyze only the scenario where $\left.E_{T, \text { rel }}\right]_{x=0.5}$ is the minimum energy consumption in relaying mode, then $\min \left(E_{T, r e l}\right)$ is less than $E_{m, d i r}$ when:

$$
\begin{gathered}
\left.E_{T, r e l}\right]_{x=0.5}<E_{m, d i r}, \\
\frac{2 A_{1}}{B_{1}-\log _{2}\left(0.5 d_{m, b}\right)}<\frac{A_{0}}{B_{0}-\log _{2}\left(d_{m, b}\right)} .
\end{gathered}
$$

Considering $A_{0}=A_{1}$ and $B_{0}=B_{1}$ (i.e. $P_{m}=P_{u}, W_{m}=$ $W_{u}, P_{m, T}=P_{u, T}$, and $\left.P_{m, R}=P_{u, R}\right)$, then

$$
\begin{gathered}
\frac{2 A_{1}}{B_{1}-\log _{2}\left(0.5 d_{m, b}\right)}<\frac{A_{1}}{B_{1}-\log _{2}\left(d_{m, b}\right)}, \\
d_{m, b}>2^{B_{1}-1} .
\end{gathered}
$$

From (19), $B_{1}=\frac{1}{\gamma} \log _{2}\left(\frac{K P_{u}}{N_{0} W_{u}}\right)$ then we have

$$
d_{m, b}>\frac{1}{2}\left(\frac{K P_{u}}{N_{0} W_{u}}\right)^{1 / \gamma} .
$$

Therefore, (47) is a condition so that the minimum global energy consumption in relaying mode is less than in direct mode.

\section{Simulation Results}

Using the simulation parameters specified in Table I in (30) we deduce that $\left.E_{T, r e l}\right]_{x=0.5}$ is a local maximum when $d_{m, b}<$ 346 meters and is a local minimum when $d_{m, b}>346$ meters. From (38), we have $\Delta \approx 78$. Then, from (40) and (41), we have $\left.\min E_{T, \text { rel }}=E_{T, \text { rel }}\right]_{x=0.5}$ if $d_{m, b}<1$ meter or $d_{m, b}>$ 548 meters, and $\left.\min E_{T, r e l}=E_{T, r e l}\right]_{x=1}$ if $1<d_{m, b}<548$ meters.

Finally, we compare the above results deduced analytically with a simulation performed using MATLAB. In Fig. 4, we plot the global energy consumption in relaying mode as a function of the distance $d_{u, b}$ normalized $\left(d_{u, b} / d_{m, b}\right)$ when the BS, the UE, and the MTD are aligned. We compare the behavior of $E_{T, r e l}$ for $d_{m, b}=\{200,400,600,800,1000\}$ meters. As shown in this figure, $E_{T, \text { rel }}$ is minimum at $x=$ 0.5 for $d_{m, b}=\{600,800,1000\}$ meters.

Furthermore, in a scenario where the BS, the UE, and the MTD are aligned, we compare the global energy consumption in direct mode and relaying mode. Simulation results are shown in Fig. 5. Using the simulation parameters in (47), we estimate that the global energy consumption in relaying mode
TABLE I

SimULATION PARAMETERS

\begin{tabular}{|c|c|}
\hline Parameter & Assumption \\
\hline Carrier Frequency $\left(f_{c}\right)$ & $900 \mathrm{MHz}$ \\
\hline MTD transmission power $\left(P_{m}\right)$ & $23 \mathrm{dBm}$ \\
\hline UE transmission power $\left(P_{u}\right)$ & $23 \mathrm{dBm}$ \\
\hline Packet Size $(L)$ & $200 \mathrm{bytes}$ \\
\hline UE power consumption in Tx state $\left(P_{u, T}\right)$ & $545 \mathrm{~mW}$ \\
\hline UE power consumption in Rx state $\left(P_{u, R}\right)$ & $90 \mathrm{~mW}$ \\
\hline MTD power consumption in Tx state $\left(P_{m, T}\right)$ & $545 \mathrm{~mW}$ \\
\hline MTD power consumption in Rx state $\left(P_{m, R}\right)$ & $90 \mathrm{~mW}$ \\
\hline MTD Bandwidth $\left(W_{m}\right)$ & $1.4 \mathrm{MHz}$ \\
\hline UE Bandwidth $\left(W_{u}\right)$ & $1.4 \mathrm{MHz}$ \\
\hline Noise Power Spectrum Density $\left(N_{0}\right)$ & $-174 \mathrm{dBm} / \mathrm{Hz}$ \\
\hline Cell radius $\left(R_{c e l l}\right)$ & $1000 \mathrm{~meters}$ \\
\hline Path loss exponent $(\gamma)$ & 3.67 \\
\hline Constant path loss factor ${ }^{1}(K)$ & 0.0070 \\
\hline
\end{tabular}

${ }^{1}$ Transmitter-Receiver distance in meters.

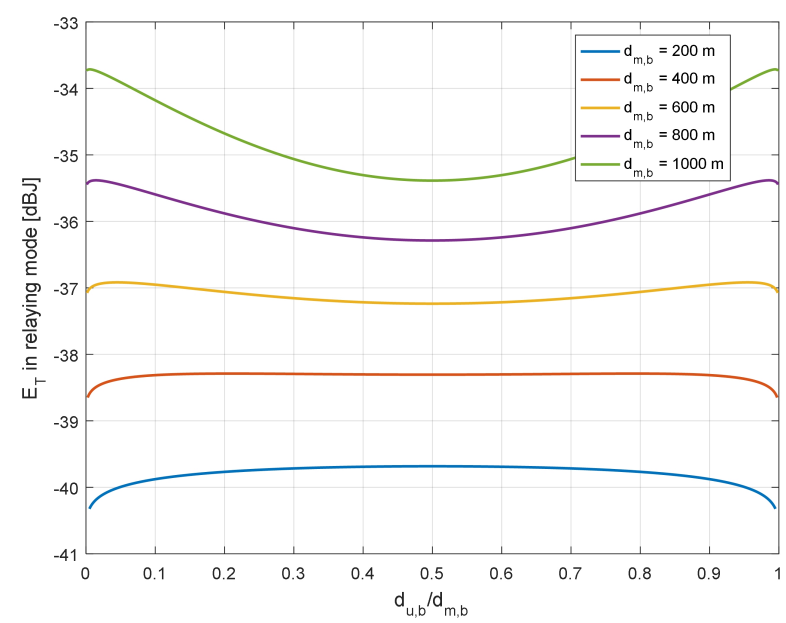

Fig. 4. $E_{T, r e l}$ as a function of the distance $d_{u, b}$ normalized, considering $\mathrm{BS}$, UE and MTD aligned.

can be less than in direct mode when $d_{m, b}>640$ meters. This condition deduced analytically can be verified by simulation.

So far, our work has been focused on searching a point in the cell where the global energy consumption in relaying mode is the lowest possible. However, in a realistic scenario, it is difficult or even impossible to find a UE located just at that point since UEs are distributed randomly in the cell. Therefore, we calculate the global energy consumption by sub-regions. We determine in which sub-regions the energy consumption is the lowest with a probability $\mathcal{P}$ of finding one or more UEs in these sub-regions. We use MATLAB to calculate the global energy consumption $E_{T, \text { rel }}$ in the whole cell, according to expression (14) and considering the simulation parameters given in Table I. To calculate $E_{T, \text { rel }}$ depending on the relay location $\left(x_{u}, y_{u}\right)$, we follow these steps:

1) Subdivide the plane into sub-regions (small squares)

2) For each sub-region, we calculate the $E_{T, \text { rel }}$ considering a relay in the center of this sub-region.

Fig. 6 shows the global energy consumption when $d_{m, b}=$ 


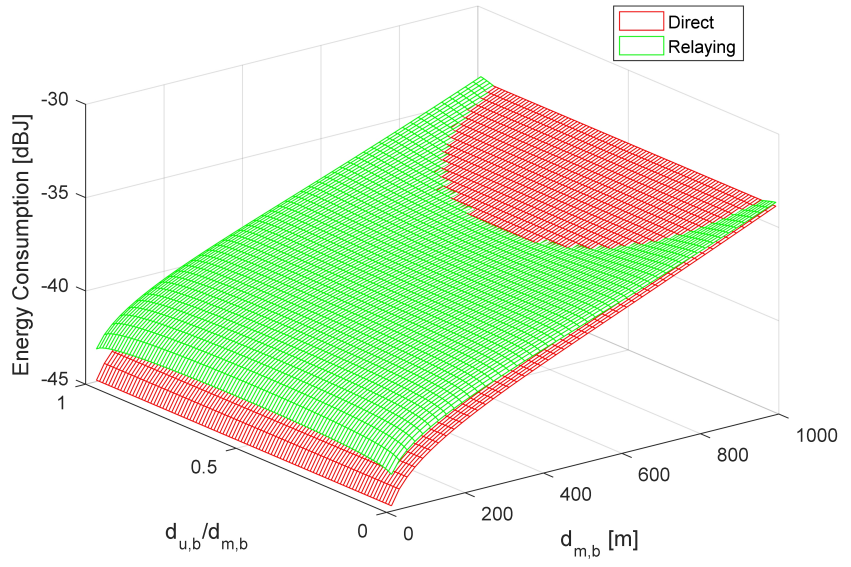

Fig. 5. Energy consumption in direct versus relaying mode as a function of the normalized distance $d_{u, b} / d_{m, b}$ and $d_{m, b}$, considering that the $\mathrm{BS}$, the $\mathrm{UE}$, and the MTD are aligned.

800 meters. We also calculate the energy consumption in direct mode $(=-35.74 \mathrm{dBJ})$ which is represented by a white oval in this figure. The sub-region where the global energy consumption is the lowest is located at the midpoint between the BS and the MTD as shown in the figure. Furthermore, as we have determined when $d_{m, b}>640$ meters, the energy consumption in relaying mode may be less than in direct mode in some sub-regions.

In Fig. 6, the energy consumption in relaying mode would be less than in direct mode if there were at least one relay within the area enclosed by the white oval. We remark using simulations for different $d_{m, b}$ values that this area tends to increase when $d_{m, b}$ increases, so the probability of finding a relay within this area will also increase as a function of $d_{m, b}$. Using a nearby UE as a relay, from an MTD point of view implies the reduction of the MTD energy consumption. However, from a global point of view (MTD and relay), the global energy consumption could be reduced only if a relay is selected appropriately. An optimal selection of relay depends on the location of the MTD and the location of the relay.

\section{CONCLUSiOn AND FUture WORKS}

In this paper, we have analyzed the behavior of energy consumption in relaying mode depending on the distance between the transmitter, the relay, and the base station. Furthermore, we have shown the interest of a D2D relay mechanism compared with a direct link with an unfavorable link budget (MTD far from the base station).

An important future work is to extend the energy consumption model considering the power control and the synchronization phase (discovery process). We also plan to consider a cost function weighting the global energy consumption model.

\section{REFERENCES}

[1] Cisco Visual Networking Index Global Mobile Data Traffic Forecast Update, 20162021, White Paper, Feb. 2017.

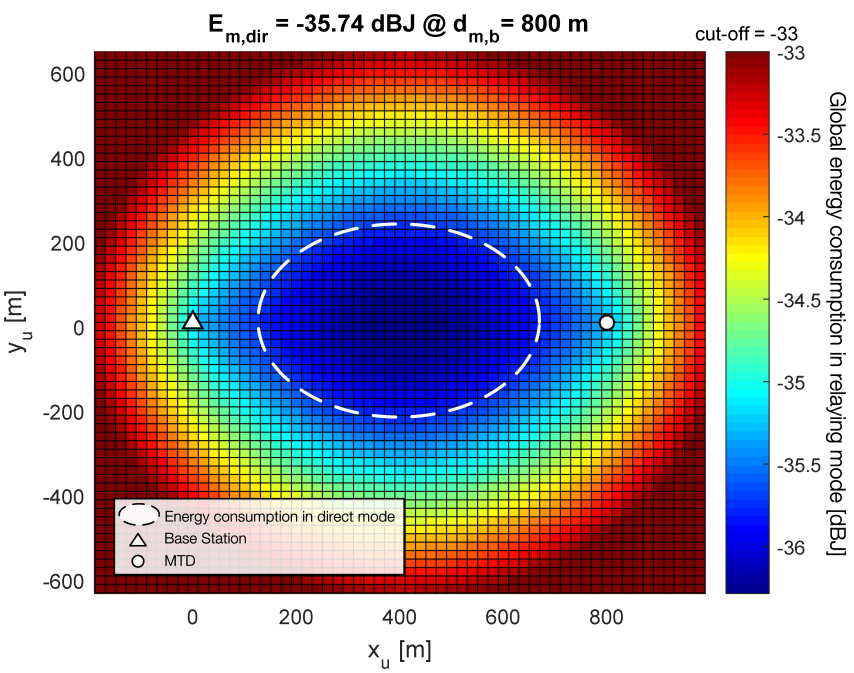

Fig. 6. Global energy consumption depending on the UE location, for $d_{m, b}=$ 800 meters.

[2] Ericsson, "5G Radio access Capabilities and technologies," White Paper, April 2016.

[3] M. R. Palattella et al., "Internet of things in the 5G Era: Enablers, Architecture, and Business Models," in IEEE Journal on Selected Areas in Communications, vol. 34, no. 3, pp. 510527, March 2016.

[4] A. Asadi, Q. Wang and V. Mancuso, "A Survey on Device-to-Device Communication in Cellular Networks," in IEEE Communications Surveys and Tutorials, vol. 16, no. 4, pp. 1801-1819, Fourthquarter 2014.

[5] C. Y. Tu, C. Y. Ho and C. Y. Huang, "Energy-Efficient Algorithms and Evaluations for Massive Access Management in Cellular Based Machine to Machine Communications," 2011 IEEE Vehicular Technology Conference (VTC Fall), San Francisco, CA, 2011, pp. 1-5.

[6] G. Miao, A. Azari, and T. Hwang, " $E^{2}$-MAC: Energy efficient medium access for massive M2M communications," IEEE Trans. Commun.,vol. 64, no. 11, pp. 47204735, Nov. 2016.

[7] G. Rigazzi, N. K. Pratas, P. Popovski, and R. Fantacci, "Aggregation and trunking of M2M traffic via D2D connections,"2015 IEEE International Conference on Communications (ICC), London, 2015, pp. 2973-2978.

[8] N. K. Pratas and P. Popovski, "Underlay of low-rate machine-type D2D links on downlink cellular links," 2014 IEEE International Conference on Communications Workshops (ICC), Sydney, NSW, 2014, pp. 423428.

[9] G. Rigazzi, F. Chiti, R. Fantacci, and C. Carlini, "Multi-hop D2D networking and resource management scheme for M2M communications over LTE-A systems," 2014 International Wireless Communications and Mobile Computing Conference (IWCMC), Nicosia, 2014, pp. 973-978.

[10] Q. Wang, M. Hempstead and W. Yang, "A Realistic Power Consumption Model for Wireless Sensor Network Devices," 2006 3rd Annual IEEE Communications Society on Sensor and Ad Hoc Communications and Networks, Reston, VA, 2006, pp. 286-295.

[11] B. Martinez, M. Montn, I. Vilajosana and J. D. Prades, "The Power of Models: Modeling Power Consumption for IoT Devices," in IEEE Sensors Journal, vol. 15, no. 10, pp. 5777-5789, Oct. 2015.

[12] "Cellular system support for ultra low complexity and low throughput internet of things," Tech. Rep. 45.820 v13.1.0, 3GPP TSG GERAN, 2015.

[13] M. Condoluci, L. Militano, A. Orsino, J. Alonso-Zarate and G. Araniti, "LTE-direct vs. WiFi-direct for machine-type communications over LTE-A systems," 2015 IEEE 26th Annual International Symposium on Personal, Indoor, and Mobile Radio Communications (PIMRC), Hong Kong, 2015, pp. 2298-2302.

[14] D. Della Penda, L. Fu and M. Johansson, "Mode selection for energy efficient D2D communications in dynamic TDD systems," 2015 IEEE International Conference on Communications (ICC), London, 2015, pp. 5404-5409. 